\title{
CORRECTION
}

\section{Correction to: Asthma and Chronic Obstructive Pulmonary Disease Overlap According to the Japanese Respiratory Society Diagnostic Criteria: The Prospective, Observational ACO Japan Cohort Study}

Shu Hashimoto (1) - Ryoko Sorimachi · Tatsunori Jinnai ·

Masakazu Ichinose

Published online: January 6, 2022

(c) Springer Healthcare Ltd., part of Springer Nature 2021

Correction to: Adv Ther (2021) 38:1168-1184

https://doi.org/10.1007/s12325-020-01573-x

In the original article, Tables 5 and 6 were published incorrectly. The correct Tables 5 and 6 are given below.

In addition, there is an error in the discussion section. The second paragraph of the discussion section currently reads: "The data obtained at registration showed that ICS use

was relatively common in Japanese ACO patients $(76.5 \%)$ compared with $36.3 \%$ of nonACO patients." This sentence should read: "The data obtained at registration showed that ICS use was relatively common in Japanese ACO patients (74.3\%) compared with $35.6 \%$ of nonACO patients."

The original article can be found online at https://doi. org/10.1007/s12325-020-01573-X.

S. Hashimoto $(\square)$

Nihon University, Itabashi-ku, Tokyo, Japan

e-mail: hashimoto.shu@nihon-u.ac.jp

S. Hashimoto

Hibiya Kokusai Clinic, Chiyoda-ku, Tokyo, Japan

R. Sorimachi · T. Jinnai

Medical Department, AstraZeneca K.K., Kita-ku,

Osaka, Japan

M. Ichinose $(\varangle)$

Academic Center of Osaki Citizen Hospital, Miyagi,

Japan

e-mail: ichinose@h-osaki.jp 
Table 5 Characteristics of patients with or without ACO at registration among those who had the data necessary for ACO diagnosis

\begin{tabular}{|c|c|c|c|c|}
\hline & \multicolumn{3}{|c|}{ Patients with the data necessary for ACO diagnosis } & \multirow{2}{*}{$\begin{array}{l}p \text { value } \\
\text { (ACO } \\
\text { vs non-ACO) }\end{array}$} \\
\hline & $\begin{array}{l}\text { All } \\
(n=396)\end{array}$ & $\begin{array}{l}\text { ACO } \\
(n=101)\end{array}$ & $\begin{array}{l}\text { Non-ACO } \\
(n=295)\end{array}$ & \\
\hline Sex, male, $n(\%)$ & $353(89.1)$ & $90(89.1)$ & $263(89.2)$ & 0.990 \\
\hline Age, years, mean (SD) & $73.2(8.0)$ & $71.5(9.6)$ & $73.8(7.3)$ & 0.025 \\
\hline BMI, $\mathrm{kg} / \mathrm{m}^{2}$, mean $(\mathrm{SD})$ & $23.0(3.4)$ & $23.0(3.1)$ & $23.0(3.5)$ & 0.869 \\
\hline \multicolumn{5}{|l|}{ Smoking status, $n$ (\%) } \\
\hline Current smoker & $42(10.6)$ & $6(5.9)$ & $36(12.2)$ & 0.108 \\
\hline Never smoker & $4(1.0)$ & $2(2.0)$ & $2(0.7)$ & \\
\hline Ex-smoker & $350(88.4)$ & $93(92.1)$ & $257(87.1)$ & \\
\hline Smoking history (pack-years) & $n=392$ & $n=99$ & $n=293$ & \\
\hline Mean (SD) & $55.1(35.8)$ & $57.7(40.7)$ & $54.2(34.1)$ & 0.441 \\
\hline Duration of COPD, years & $n=390$ & $n=99$ & $n=291$ & \\
\hline Mean (SD) & $5.3(5.4)$ & $5.3(4.6)$ & $5.3(5.7)$ & 0.997 \\
\hline \multicolumn{5}{|l|}{ GOLD stage, $n(\%)$} \\
\hline I & $125(31.6)$ & $30(29.7)$ & $95(32.2)$ & 0.207 \\
\hline II & $194(49.0)$ & $54(53.5)$ & $140(47.5)$ & \\
\hline III & $59(14.9)$ & $16(15.8)$ & $43(14.6)$ & \\
\hline IV & $18(4.5)$ & $1(1.0)$ & $17(5.8)$ & \\
\hline \multicolumn{5}{|l|}{ Complication/comorbidity, $n(\%)$} \\
\hline Asthma (based on physician's diagnosis) & $177(44.7)$ & $68(67.3)$ & $109(36.9)$ & $<0.001$ \\
\hline \multirow[t]{2}{*}{ Age at onset $<40$ years } & $n=175$ & $n=67$ & $n=108$ & \\
\hline & $24(13.7)$ & $23(34.3)$ & $1(0.9)$ & $<0.001$ \\
\hline \multirow{2}{*}{ Age at onset $\geq 40$ years } & $n=175$ & $n=67$ & $n=108$ & \\
\hline & $151(86.3)$ & $44(65.7)$ & $107(99.1)$ & $<0.001$ \\
\hline Allergic rhinitis & $57(14.4)$ & $37(36.6)$ & $20(6.8)$ & $<0.001$ \\
\hline Heart failure & $45(11.4)$ & $3(3.0)$ & $42(14.2)$ & 0.002 \\
\hline Diabetes & $58(14.6)$ & $15(14.9)$ & $43(14.6)$ & 0.946 \\
\hline Respiratory medication at baseline, $n(\%)$ & $n=396$ & $n=101$ & $n=295$ & \\
\hline ICS (monotherapy or any combinations) & $180(45.5)$ & $75(74.3)$ & $105(35.6)$ & $<0.001$ \\
\hline ICS monotherapy & $4(1.0)$ & $3(3.0)$ & $1(0.3)$ & 0.053 \\
\hline LAMA monotherapy & $44(11.1)$ & $10(9.9)$ & $34(11.5)$ & 0.654 \\
\hline LABA monotherapy & $20(5.1)$ & $1(1.0)$ & $19(6.4)$ & 0.031 \\
\hline LAMA and $\mathrm{LABA}^{\mathrm{a}}$ & $138(34.8)$ & $11(10.9)$ & $127(43.1)$ & $<0.001$ \\
\hline ICS and LABA ${ }^{a}$ & $65(16.4)$ & $22(21.8)$ & $43(14.6)$ & 0.092 \\
\hline
\end{tabular}


Table 5 continued

\begin{tabular}{|c|c|c|c|c|}
\hline & \multicolumn{3}{|c|}{ Patients with the data necessary for ACO diagnosis } & \multirow{2}{*}{$\begin{array}{l}p \text { value } \\
\text { (ACO } \\
\text { vs non-ACO) }\end{array}$} \\
\hline & $\begin{array}{l}\text { All } \\
(n=396)\end{array}$ & $\begin{array}{l}\text { ACO } \\
(n=101)\end{array}$ & $\begin{array}{l}\text { Non-ACO } \\
(n=295)\end{array}$ & \\
\hline ICS and LAMA & $4(1.0)$ & $2(2.0)$ & $2(0.7)$ & 0.270 \\
\hline ICS, LAMA, and LABA ${ }^{a}$ & $107(27.0)$ & $48(47.5)$ & $59(20.0)$ & $<0.001$ \\
\hline \multicolumn{5}{|l|}{ Symptoms based on interview } \\
\hline Presence of variable symptoms, $n$ (\%) & $96(24.2)$ & $67(66.3)$ & $29(9.8)$ & $<0.001$ \\
\hline \multirow[t]{2}{*}{ Presence of paroxysmal symptoms, $n$ (\%) } & $n=387$ & $n=95$ & $n=292$ & \\
\hline & $90(23.3)$ & $57(60.0)$ & $33(11.3)$ & $<0.001$ \\
\hline \multicolumn{5}{|l|}{ Questionnaire } \\
\hline CAT & $n=395$ & $n=101$ & $n=294$ & \\
\hline Mean (SD) & $10.3(7.0)$ & $10.7(7.8)$ & $10.1(6.7)$ & 0.492 \\
\hline mMRC & $n=395$ & $n=101$ & $n=294$ & \\
\hline Mean (SD) & $1.0(1.0)$ & $1.0(1.0)$ & $1.1(1.0)$ & 0.523 \\
\hline ACQ & $n=394$ & $n=101$ & $n=293$ & \\
\hline Mean (SD) & $0.5(0.8)$ & $0.9(1.0)$ & $0.4(0.6)$ & $<0.001$ \\
\hline
\end{tabular}

$A C O$ asthma-COPD overlap, $A C Q$ asthma control questionnaire, $B M I$ body mass index, $C A T$ COPD assessment test, COPD chronic obstructive pulmonary disease, GOLD Global Initiative for Chronic Obstructive Lung Disease, ICS inhaled corticosteroids, $L A B A$ long-acting $\beta_{2}$-agonist, $L A M A$ long-acting muscarinic antagonist, $m M R C$ modified Medical Research Council questionnaire, $S D$ standard deviation

${ }^{a}$ Including any combinations of separate and combined inhalers

Table 6 Biomarker and lung function measurements of patients with or without ACO at registration among those who had the data necessary for ACO diagnosis

\begin{tabular}{|c|c|c|c|c|}
\hline & \multicolumn{3}{|c|}{ Patients with the data necessary for ACO diagnosis } & \multirow{2}{*}{$\begin{array}{l}p \text { value } \\
\text { (ACO vs } \\
\text { non-ACO) }\end{array}$} \\
\hline & $\begin{array}{l}\text { All } \\
(n=396)\end{array}$ & $\begin{array}{l}\text { ACO } \\
(n=101)\end{array}$ & $\begin{array}{l}\text { Non-ACO } \\
(n=295)\end{array}$ & \\
\hline \multicolumn{5}{|l|}{ Biomarkers } \\
\hline FeNO, ppb & $n=355$ & $n=86$ & $n=269$ & \\
\hline Mean (SD) & $27.4(23.5)$ & $47.8(35.5)$ & $20.9(12.5)$ & $<0.001$ \\
\hline Median & 22.0 & 41.5 & 20.0 & \\
\hline $\mathrm{FeNO}>35 \mathrm{ppb}, n(\%)$ & $75(21.1)$ & $59(68.6)$ & $16(5.9)$ & $<0.001$ \\
\hline $\begin{array}{l}\text { Peripheral eosinophils absolute } \\
\text { count, cells } / \mu \mathrm{L}\end{array}$ & $n=370$ & $n=92$ & $n=278$ & \\
\hline Mean (SD) & $241.1(277.9)$ & $392.8(472.2)$ & $190.9(139.4)$ & $<0.001$ \\
\hline Median & 176.0 & 289.5 & 160.5 & \\
\hline Eosinophil ratio, \% & $n=370$ & $n=92$ & $n=278$ & \\
\hline
\end{tabular}


Table 6 continued

\begin{tabular}{|c|c|c|c|c|}
\hline & \multicolumn{3}{|c|}{ Patients with the data necessary for ACO diagnosis } & \multirow{2}{*}{$\begin{array}{l}p \text { value } \\
(\mathrm{ACO} \text { vs } \\
\text { non-ACO) }\end{array}$} \\
\hline & $\begin{array}{l}\text { All } \\
(n=396)\end{array}$ & $\begin{array}{l}\text { ACO } \\
(n=101)\end{array}$ & $\begin{array}{l}\text { Non-ACO } \\
(n=295)\end{array}$ & \\
\hline Mean (SD) & $3.8(3.8)$ & $5.9(6.0)$ & $3.1(2.3)$ & $<0.001$ \\
\hline Total $\mathrm{IgE}, \mathrm{IU} / \mathrm{mL}$ & $n=278$ & $n=75$ & $n=203$ & \\
\hline Mean (SD) & $415.1(837.9)$ & $833.2(1330.2)$ & $260.6(475.1)$ & $<0.001$ \\
\hline Median & 133.5 & 398.0 & 92.0 & \\
\hline Presence of $\geq 1$ positive allergen & $n=147$ & $n=39$ & $n=108$ & \\
\hline detected in the specific $\operatorname{IgE}$ exam, $n(\%)$ & $64(43.5)$ & $24(61.5)$ & $40(37.0)$ & 0.008 \\
\hline \multicolumn{5}{|l|}{ Lung function } \\
\hline Post-bronchodilator $\mathrm{FEV}_{1} / \mathrm{FVC} \%$, mean $(\mathrm{SD})$ & $53.1(12.0)$ & $53.3(9.9)$ & $53.0(12.7)$ & 0.792 \\
\hline Post-bronchodilator $\% \mathrm{FEV}_{1}$ predicted, mean $(\mathrm{SD})$ & $68.1(21.7)$ & $69.2(20.6)$ & $67.7(22.1)$ & 0.545 \\
\hline $\begin{array}{l}\mathrm{FEV}_{1} \text { reversibility (change in } \mathrm{FEV}_{1} \\
>12 \% \text { and }>200 \mathrm{~mL} \text { ) }\end{array}$ & $n=239$ & $n=61$ & $n=178$ & \\
\hline Yes, $n(\%)$ & $14(5.9)$ & $8(13.1)$ & $6(3.4)$ & 0.010 \\
\hline \multirow[t]{2}{*}{ Presence of emphysema on CT, $n(\%)$} & $n=311$ & $n=80$ & $n=231$ & \\
\hline & $265(85.2)$ & $64(80.0)$ & $201(87.0)$ & 0.128 \\
\hline \multirow[t]{2}{*}{$\% D_{\mathrm{LCO}}<80 \%$ or $\% D_{\mathrm{LCO}} / V_{\mathrm{A}}<80 \%, n(\%)$} & $n=183$ & $n=53$ & $n=130$ & \\
\hline & $130(71.0)$ & $31(58.5)$ & $99(76.2)$ & 0.017 \\
\hline
\end{tabular}

$A C O$ asthma-COPD overlap, $C T$ computed tomography, $D_{L C O}$ diffusion capacity for carbon monoxide, $\mathrm{FeNO}$ fractional exhaled nitric oxide, $F E V_{1}$ forced expiratory volume in one second, $F V C$ forced vital capacity, $I g$ immunoglobulin, $S D$ standard deviation, $V_{A}$ alveolar volume 